\title{
Ultrafast Ultrasound Imaging
}

\author{
Jeremy Bercoff \\ SuperSonic Imagine \\ France
}

\section{Introduction}

Ultrasound can be considered as a disruptive technology in the medical device arena (Christensen, 2003). A disruptive technology has the potential to break the rules of existing markets. Thanks to its real time capabilities, its non ionizing properties and its cost - much lower than any other medical modality - ultrasound has significantly impacted clinical segments within radiology, obstetrics, vascular or cardiology and created new markets of emergency medicine and intervention. It could, in the future, change the rules for screening (where whole breast ultrasound devices are entering the market of breast imaging), diagnosis (with the standardization of elastography techniques in the prostate) and surgery (with HIFU - High Intensity Focused Ultrasound -, histotripsy devices and therapy monitoring tools).

In this context, innovations in the ultrasound field always have enormous potential. In the history of ultrasound, many innovations have been developed since its establishment as a medical imaging device in the 1960s, roughly one or two per decade (Szabo, 2004). The key innovation that launched the modality in the 1960s, is the real time imaging capability through mechanical scanning. Multichannel systems with electronic control of transducer arrays were developed in the 1970s. In the 1980s, flow analysis tools came to maturity through color flow imaging and quantitative Doppler modes (Pulse Wave Doppler PWD). In the 1990's significant improvements in image quality were made possible with the introduction of real time compounding techniques and harmonic imaging. Although many of these concepts were studied in research laboratories years before the commercial dates cited above, it is systematically the maturity of a new technology that trigger the introduction of the innovations on commercially available platforms: for example, real time imaging was triggered by microprocessors development, Doppler modes were prompted by digital signal processing chips with enough dynamics to detect, at the same time, very weak blood signal and strong tissue echoes. The introduction of low cost Analog to Digital (A/D) converters has led to fully digital systems, significantly increasing the quality of the information delivered. Harmonic imaging was triggered by large bandwidth transducers, allowing reception of the signal at twice the transmit frequency.

In the first decade of the 21st century, technology moved towards extensive miniaturization leading to the introduction of high performances portable devices. Portable devices have created new markets for ultrasound - the emergency market for example, underlying again the disruptive potential of the modality. Today portable devices are the primary sources of 
market growth in the industry and miniaturization can be considered as a global trend of the ultrasound industry: available technologies and innovations are progressively integrated in portable systems.

The figure below summarizes the evolution of ultrasound in the last decades.

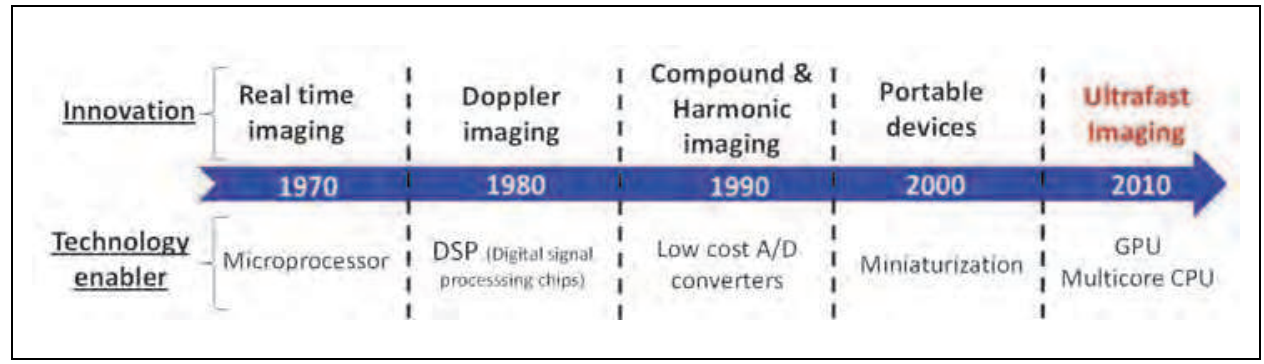

Fig. 1. A few important innovations in ultrasound imaging and their corresponding technology enablers.

Today a new technological breakthrough is ongoing with the advent of massive parallel computing capabilities. This results from the incredible demand in processing and display performances needed in the videogame industry. In addition to multicore architecure CPU's, new graphical processing units (GPU) allow parallel processing on thousands of channels simultaneously. This technology is available for the ultrasound industry and is the enabler to full software-based architecture systems. In 2009, SuperSonic Imagine introduced the first full software-based ultrasound system (Aixplorer ${ }^{\circledR}$ ): instead of increasing integrated hardware processing channels, all the processing is performed by the software unit (CPU and GPUs). The concept of processing channels disappears - the system is able to compute in parallel as many channels as required by the acquisition.

This architecture paves a new way to perform ultrasound imaging: ultrafast ultrasound imaging. This is the focus of this chapter: what is ultrafast imaging (section 2), what new information can be assessed using it (section 3), how can we revisit standard ultrasound modes using ultrafast capabilities and enhance performances of current ultrasound devices (sections 4) and what innovations could it bring in the future (section 5) ?

\section{Ultrafast ultrasound imaging: definition and example}

\subsection{Conventional ultrasound imaging}

Ultrasound imaging is usually performed by sequential insonification of the medium using focused beams. Each focused beam allows the reconstruction of one image line. A typical 2D image is made of a few tens of lines (64 to 512). The overall sequence is illustrated on Fig. 2. The frame rate of the imaging mode is set by the time required to transmit a beam, receive and process the backscattered echoes from the medium and repeat that for all the lines of the image.

For a conventional 2D image, the time to build an image is:

$$
T_{\text {image }}=\frac{N_{\text {lines }} * 2 * Z}{c}
$$




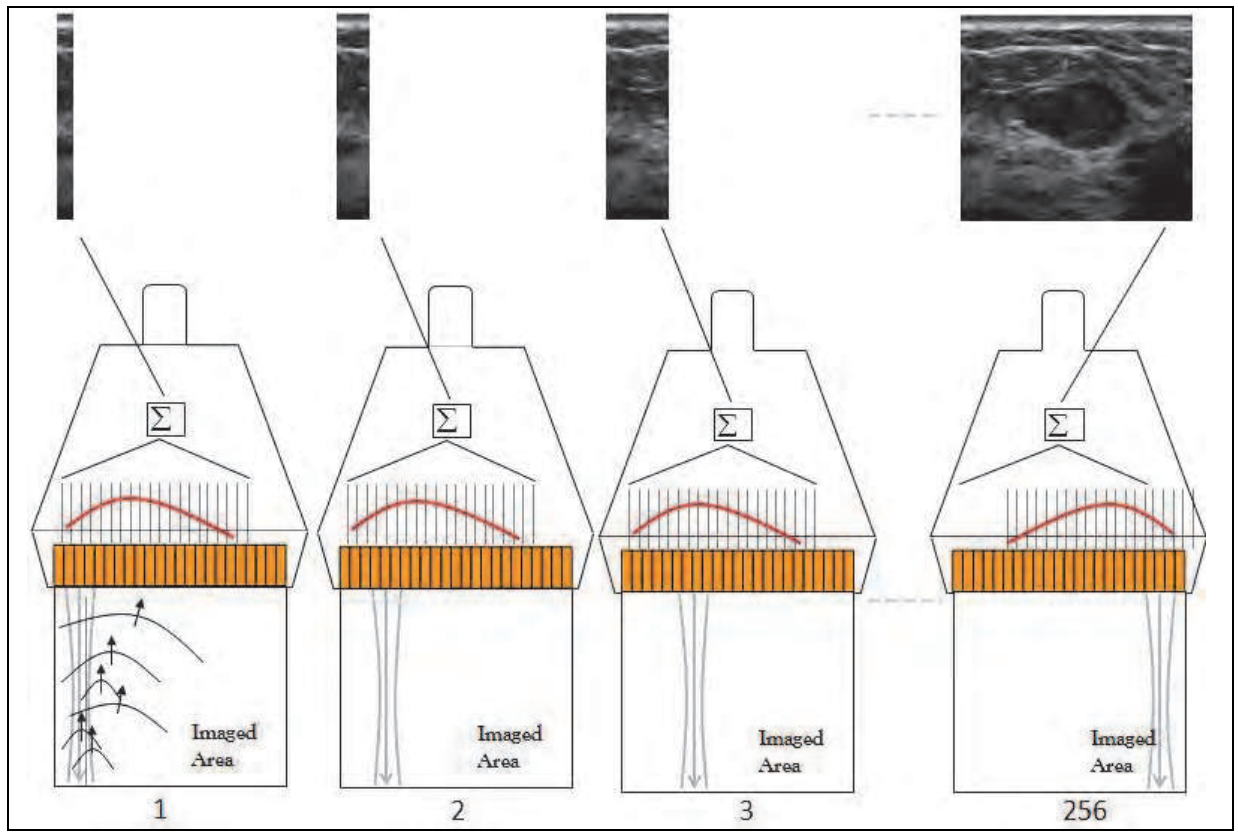

Fig. 2. Conventional imaging acquisition process

Where $\mathrm{Z}$ is the image depth, $\mathrm{c}$ the speed of ultrasound waves assumed constant $(1540 \mathrm{~m} / \mathrm{s})$ and Nlines the number of lines in the image.

The maximum frame rate that can be reached with this technique is:

$$
F R_{\max }=\frac{1}{T_{\text {image }}}
$$

For example, an image of $5 \mathrm{~cm}$ in depth and 256 lines in width would have the following frame rate:

$$
F R_{\max }=60 \mathrm{~Hz}
$$

Ultrasound system architectures were designed to process one image line at a time.

\subsection{Increasing ultrasound imaging frame rate}

Limitations of the conventional approach appears as soon as higher frame rates are required, typically in echocardiography for the heart motion analysis, as well as in 3D/4D imaging where the number of lines become significant ( $\sim$ a few thousands).

Parallelization schemes have been considered to overcome these limitations. In the academic area this has been reported as soon as the late 70's (Delannoy, 1979; Shattuck, 1984; Von Ramm, 1991). Most current systems have multiline capabilities: for each transmit beam, several lines (typically from 2 to 16) are computed. Multiline processing can be used either to increase the frame rate (for echocardiography for example) either to increase the number of lines computed per image (for 3D imaging). 


\subsection{Ultrafast imaging}

With or without multiline capabilities, current ultrasound systems are built on a serialized architecture and images are reconstructed sequentially from several equivalent transmits.

Ultrafast imaging breaks this paradigm. An ultrafast imaging system is able to compute in parallel as many lines as requested and is therefore capable of computing a full image from one single transmit whatever the size and the characteristics of the image. In such a system the image frame rate is no longer limited by the number of lines reconstructed but by the time of flight of a single pulse to propagate in the medium and get back to the transducer. Table 1 gives typical frame rates for different ultrasound clinical applications using conventional and ultrafast architectures.

\begin{tabular}{|c|c|c|c|}
\hline Application & $\begin{array}{c}\text { Typical imaging } \\
\text { depth }\end{array}$ & $\begin{array}{c}\text { Conventional } \\
\text { architecture }\end{array}$ & Ultrafast architecture \\
\hline Abdominal imaging & $20 \mathrm{~cm}$ & $20 \mathrm{~Hz}$ & $3800 \mathrm{~Hz}$ \\
\hline Cardiac Imaging & $15 \mathrm{~cm}$ & $150 \mathrm{~Hz}$ & $5000 \mathrm{~Hz}$ \\
\hline Breast imaging & $5 \mathrm{~cm}$ & $60 \mathrm{~Hz}$ & $15000 \mathrm{~Hz}$ \\
\hline
\end{tabular}

Table 1. Example of typical frame rates in different clinical applications for conventional and ultrafast architectures.

New applications of ultrafast systems have been reported in the literature. Fink demonstrated for the first time that transient shear waves, never visualized before on an ultrasound scanner, can be imaged (Sandrin, 2000). Jensen used an ultrafast device to implement synthetic imaging techniques and derive vectorial estimation of flow motion (Jensen 2005). Ultrafast prototypes reported in those works allowed the storage of acquisitions in a digital memory stack and then the transfer to a PC. Processing was then performed offline on the stored data.

Although the concept of ultrafast imaging has been explored in academics during the last decade, it is only recently that this technology has entered the commercial realm due to major technological barriers that had to be overcome.

For example, to achieve ultrafast imaging, the image computation must be performed on a fully parallelized platform, typically a software-based platform.

There are two technologically challenging aspects to building a fully software-based platform:

- the data transfer rate from the acquisition module to the processing unit. As raw (non beamformed) Radio Frequency (RF) signals are directly transferred to the PC, the data rate required to perform real time imaging is huge: several GigaBytes/s.

- the processing unit needs to be powerful enough to ensure real time imaging. As an example, conventional gray scale imaging requires 1 to 2 Gigaflops (multiplication + addition) per second.

New powerful processing units (GPUs) have reached a satisfactory level of performance at the end of the 2000s. Consequently, GPU's are more and more used in the medical field to speed up processing algorithms (Schiwietz, 2006; Xu, 2007; Rosenzweig, 2011). The ultrafast architecture leverages this processing power by combining it with fast numerical links (PCI express technology) capable of transferring huge volume of data to these units. This combination allows the shift of the beamforming process - the most demanding processing step of an ultrasound system - from hardware to software, enabling full parallelization of ultrasound image computation.

Fig. 3 represents the architecture of an ultrafast system compared to a conventional one. 


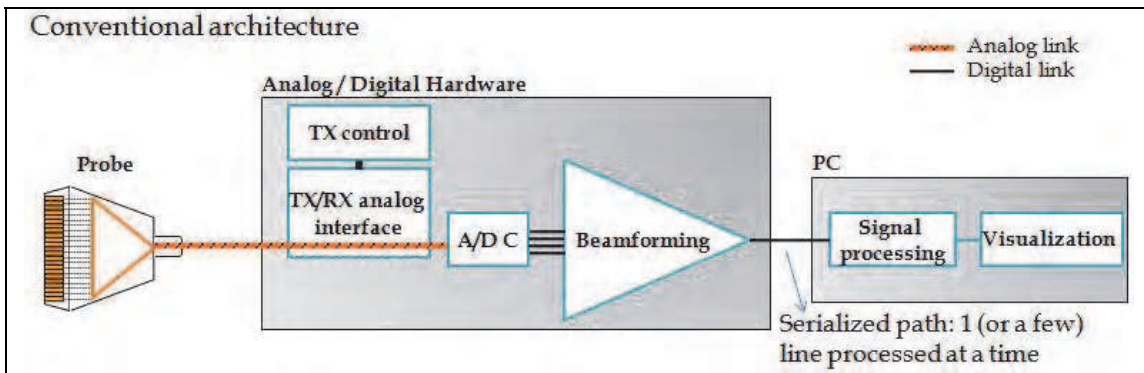

Ultrafast architecture

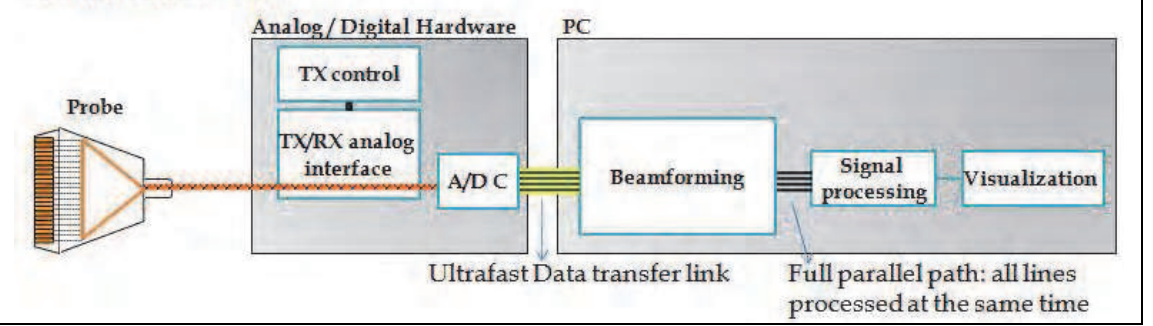

Fig. 3. As beamforming is performed in software, full parallelization of image formation can be performed. Each insonification can therefore lead to a full image. (TX refers to Transmit and RX to Receive).

\subsection{Ultrafast imaging using coherent plane wave compound}

There are many ways to leverage an Ultrafast imaging architecture (Lu, 1998; Jensen 2005). SuperSonic Imagine's approach is based on the use of plane wave insonifications. A plane wave is generated by applying flat delays on the transmit elements of the ultrasound probe as illustrated on Fig. 4. The generated wave will insonify the whole area of interest.

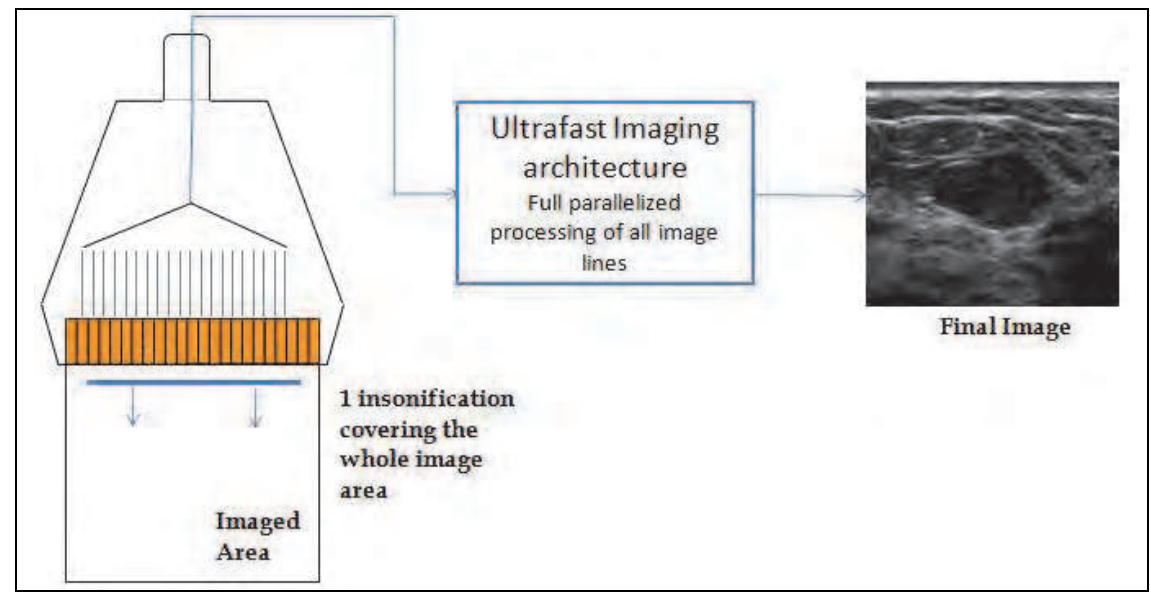

Fig. 4. A plane wave is sent by a linear transducer and insonifies the whole region of interest. An ultrasound image is computed from this single insonification. 
The backscattered echoes are then recorded and processed by the ultrafast scanner to compute an image of the insonified area.

Plane wave imaging allows the computation of one full ultrasound image per transmit at the expense of the image quality. As the transmit focalization step is removed, the image contrast and resolution are reduced, as illustrated in the figure below:

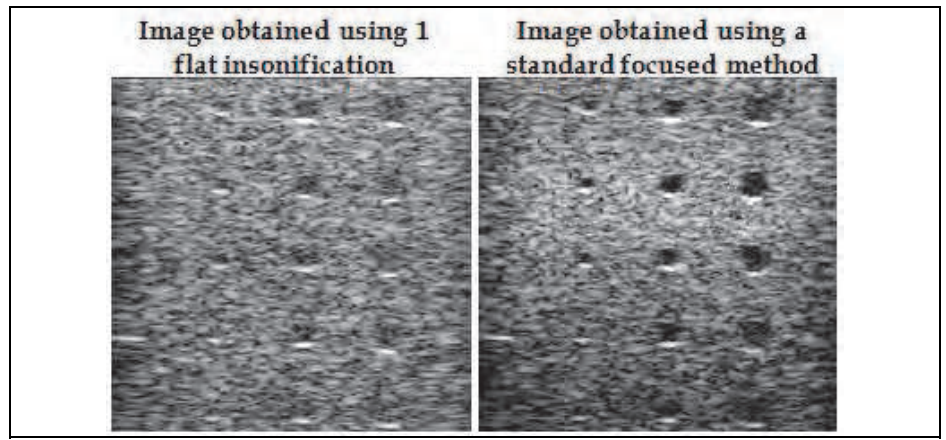

Fig. 5. Image of a phantom with anechoic inclusion of different sizes with plane wave insonification (left) and standard focused method (right). The images were acquired with a $5 \mathrm{MHz}$ linear probe.

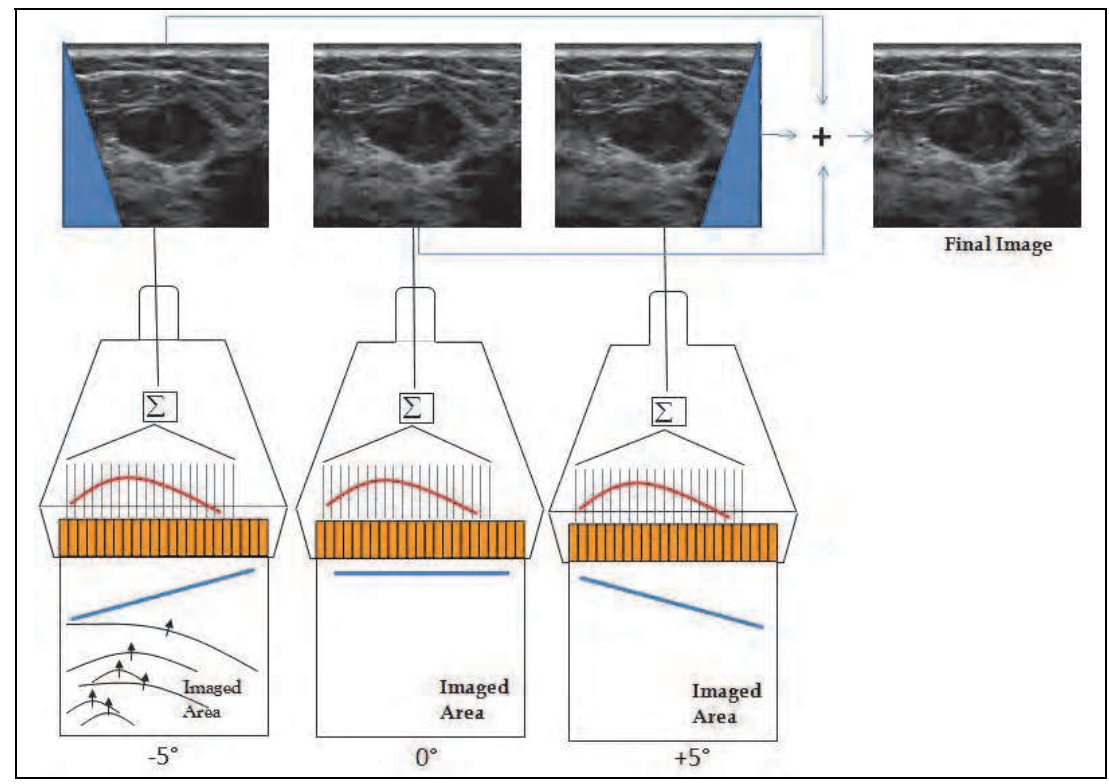

Fig. 6. Ultrasound image obtained using ultrafast coherent plane wave compound

To overcome this limitation, several tilted plane waves are sent into the medium (Montaldo, 2009) and coherently summed to compute a full image. Using this method the transmit focalization step is retrospectively done by this summation (Fig. 6). The quality of the final 
image is therefore dependent on the number of angles used to reconstruct it as illustrated on Fig. 7.

There is a trade off between the maximum ultrafast frame rate achievable by the mode and the image quality: the higher the number of angles, the better the image quality.

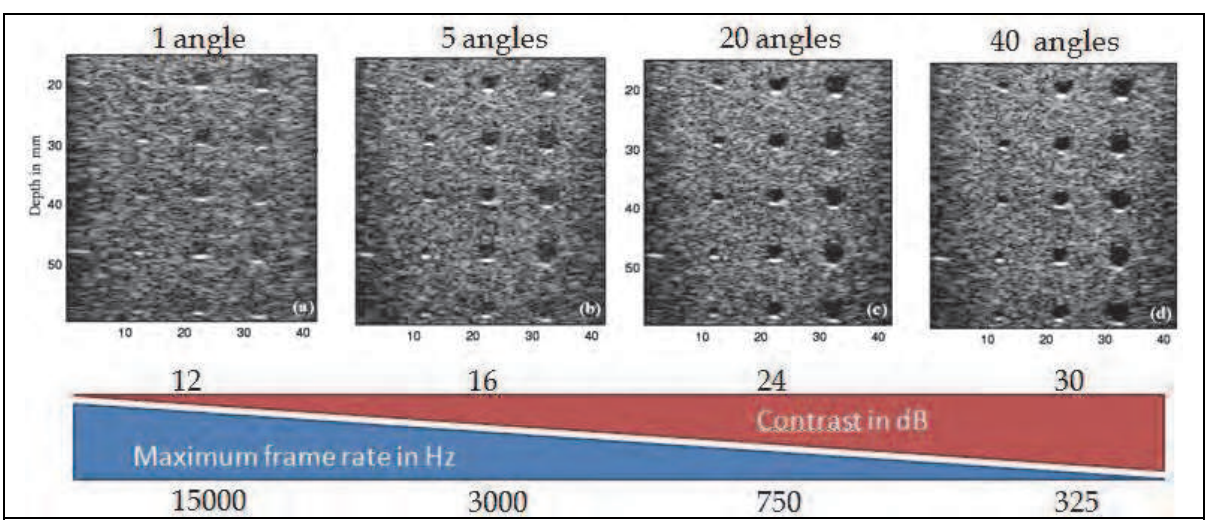

Fig. 7. Image quality as a function of the number of angles used to compute the final ultrasound image for a $40 \mathrm{~mm}$ depth image.

The ultrafast compounded acquisition sequence presents several advantages:

- Firstly, the retrospective transmit focalization can be done dynamically for each pixel of the image increasing the homogeneity of the final image compared to physical insonification.

- Secondly, the number of firings required to obtain an image of a quality equivalent to a focused mode (in terms of contrast and resolution) is around 5 to 10 times lower (Montaldo, 2009). As a consequence, frame rates of ultrasound imaging can be increased by the same factor using coherent plane wave strategies on an ultrafast system. Fig. 8 shows an example of equivalent quality ultrasound images using the coherent plane wave approach and the focused one. Maximum reachable frame rates increase from $30 \mathrm{~Hz}$ to more than $300 \mathrm{~Hz}$.

- Finally by cleverly trading off the compromises in the image quality, imaging frame rates of a few thousands of $\mathrm{Hz}$ can be reached and allow a full new range of applications and innovations.

This chapter presents two innovations that leverage ultrafast imaging on an ultrasound system: the first one is a new imaging mode called Shear Wave Elastography that provides quantitative visco-elastic analysis of tissues. The second one is a new way to perform Doppler flow analysis changing the performances and workflow paradigms of current available Color and PW modes.

\section{Shear wave elastography}

\subsection{Transient shear waves for tissue mechanical investigation}

Ultrasound imaging provides both morphological (gray scale images) and functional imaging (flow imaging) of soft tissue. Using ultrafast capabilities, a third dimension can be added to ultrasound: physio-pathological information through the assessment of tissue 


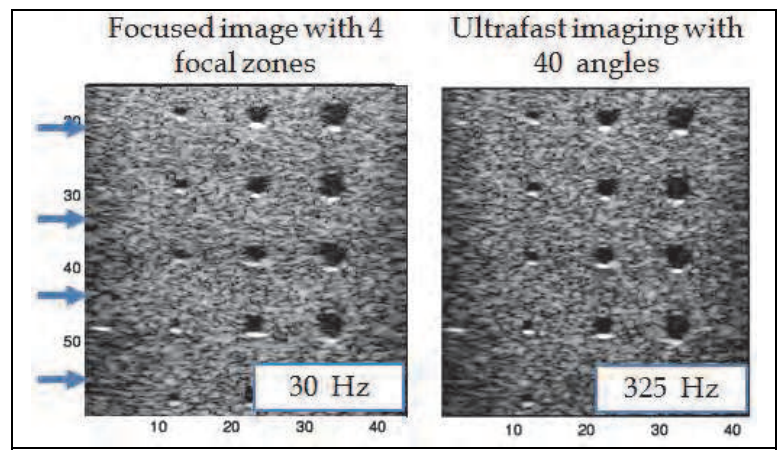

Fig. 8. 2 images of equivalent quality using focused (left) and ultrafast (right) techniques. Maximum achievable frame rates are respectively 30 and $325 \mathrm{~Hz}$.

viscoelasticity. Ultrafast imaging can be used to capture phenomena that have never been imaged on commercial ultrasound devices: transient shear waves propagating in soft tissue. Shear wave imaging leads to quantification of tissue mechanical properties.

\subsubsection{Shear waves in soft tissues}

Two types of mechanical waves propagate in soft tissue: compressional waves (ultrasound waves are compressional waves in a given frequency range) and shear waves. Compressional waves travel much faster than shear waves in soft tissue: typically 1 to $1500 \mathrm{~m} / \mathrm{s}$ compared to $10 \mathrm{~m} / \mathrm{s}$ for shear waves. In other words, the bulk modulus (K) of soft tissue is much larger than the shear modulus $(\mu)$ (a factor $10^{6}$ higher).

$$
\mathrm{K}>>\mu
$$

This has two important consequences:

- Tissue viscoelasticity is only dependent on the shear modulus. The Young's modulus, that quantifies tissue viscoelasticity, can be written :

$$
E=\frac{9 * K^{*} \mu}{3 K+\mu} \approx 3 \mu
$$

- The difference in propagation speed is so large that shear wave motion can be considered as negligible during the propagation time of a compressional wave. Imaging methods relying on compressional waves such as ultrasound can therefore be used to record propagation of shear waves. Note that this is not true in other solids such as metals or rocks (in seismology for example, bulk waves cannot image shear waves)

In summary, shear waves reflect tissue viscoelasticity properties and they can be imaged using ultrasound.

\subsubsection{Imaging shear waves: need for ultrafast imaging}

If compressional waves can propagate within tissue on a very large frequency range [up to the $\mathrm{GHz}$ ), shear waves suffer from much stronger viscous/attenuation effects. Maximum shear wave frequencies propagating in human tissue are organ-dependent and typically vary between $500 \mathrm{~Hz}$ and $2000 \mathrm{~Hz}$. As a consequence the minimum frame rate required to 
correctly sample transient waves are of a few thousands Hertz (from $1000 \mathrm{~Hz}$ to $4000 \mathrm{~Hz}$ taking the Nyquist limit).

Those frame rates are only achievable using ultrafast imaging.

In order to image shear waves, the system must be tuned to maximize the imaging frame rate. Typically a single flat wave is sent to compute a full image (Fig. 4), allowing to reach the required frame rates (a few thousands $\mathrm{Hz}$ ), the maximum value only depending on the considered image depth (time of flight of the wave back and forth from the maximum depth imaged).

\subsubsection{Generating transient shear waves}

There are three different types of source of transient shear waves in the body.

The first type is natural body vibrations: heart beating, arterial pulses or voice are examples of vibrating sources that induce shear waves. It is a free source of information but the assessment of reliable information is challenging outside of the vicinity of the vibrating organ.

To better control the generation of the vibration, external vibrators that create controlled transient pulses have been proposed. The first report of externally generated transient shear wave analysis was published in the 1990s (Catheline, 1999). A that time, ultrafast imaging was not used and shear wave propagation was analyzed along a single ultrasound line. The work was extended to 2D shear wave imaging using the first ultrafast imaging prototype (Sandrin, 2000) and the first quantitative elasticity image was shown.

Also in the late 1990s, Sarvazyan proposed a third way to generate transient shear waves in the body (Sarvazyan, 1998): the acoustic radiation force induced by ultrasound beams. If sufficient energy if applied at the focus of an ultrasound beam, tissue can be remotely pushed in the direction of the ultrasound wave propagation. A transient shear wave that propagates transversally is generated as illustrated on Fig. 9.

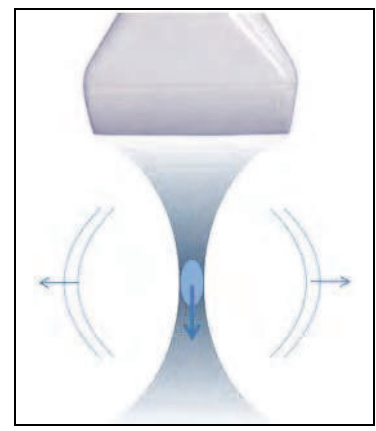

Fig. 9. Radiation force from an ultrasound focused beam generates a transverse bipolar shear wave.

In Sarvazyan's setup, the shear wave was induced with a specific transducer and the motion was recorded using a separate conventional scanner and iterative methods.

In 2004, a new imaging mode has been introduced coupling radiation force induced transient shear waves and ultrafast imaging called Supersonic Shear Imaging (Bercoff, 2004). In this approach, the shear wave is generated and imaged with the same ultrasound probe. The generation method was based on the induction of a shear wave source that moves into 
the body at a supersonic speed, allowing, through the equivalent of a sonic boom, the creation of high amplitude shear waves in human organs.

\subsubsection{Measuring tissue viscoelasticity}

Once properly generated and imaged, a transient shear wave can provide many insights on the mechanical properties of the imaged tissue. Fig. 10 illustrates the propagation of a shear wave in a tissue-mimicking phantom using Supersonic Shear Imaging. The wave is captured on a $2 \mathrm{D}$ imaging plane thanks to ultrafast imaging.

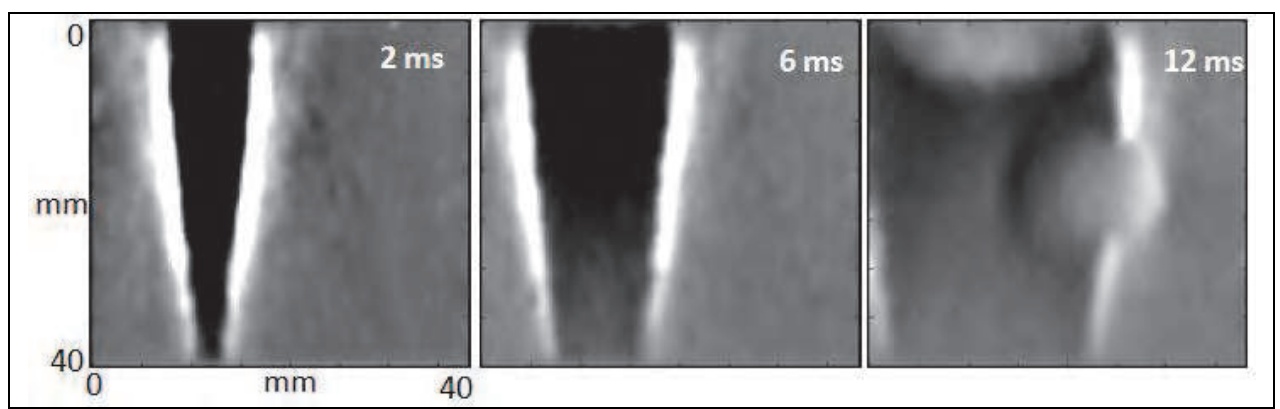

Fig. 10. 3 snapshots of a shear wavefront propagating in a phantom. The gray level indicates amplitude of the displacements generated by the shear in the tissue. The wavefront is distorted when passing through a harder inclusion (right image) as the shear wave propagates faster in the inclusion.

The phase velocity as a function of the frequency as well as the group velocity can be calculated locally.

Fig. 11 shows a group velocity map derived from the ultrafast imaging scanner in the same mimicking phantom.

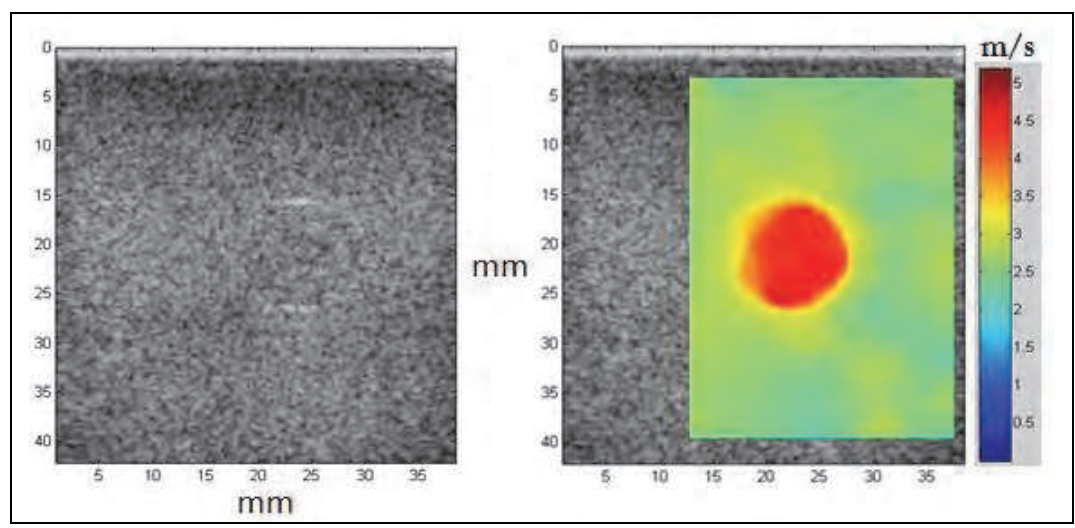

Fig. 11. Shear wave velocity map superimposed on the ultrasound image of the phantom. The harder spherical inclusion appears on the velocity map in red while it is barely visible on the ultrasound image. This enhances the fact that the contrast given by both imaging modes are uncorrelated. 
Because the shear wave is imaged inside the medium, all wave components can be assessed and used for velocity estimation including evanescent waves. The resolution of the shear wave velocity image is therefore not limited by the shear wavelength but by the wavelength of the imaging method, i.e. ultrasound. In the above example, the resolution of the image is $1 \mathrm{~mm}$ while the shear wavelength is around $15 \mathrm{~mm}$.

Depending on the organ, different rheological models can be used to derive tissue mechanical characteristics from the shear wave propagation map.

- In a purely elastic medium, phase velocity does not vary as a function of frequency (Royer, 2000) and is equal to the group velocity. The velocity is directly linked to tissue Young's modulus E through the formula where $c$ is the shear wave speed and $\rho$ the tissue density.

$$
E=3 \rho c^{2}
$$

- In a viscoelastic medium such as breast or liver, the phase velocity increases as a function of frequency. Many rheological models can be considered such as the Voigt or the thermoviscous models. Most of them appear to be consistent only in a limited frequency range (Orescanin, 2010). A well-established model for compressional waves is the time causal model (Szabo, 2004) which shows a power law dependence of the attenuation and phase velocity as a function of frequency and works on the full frequency range. It can also be applied to shear waves:

$$
\frac{1}{c(f)}=\frac{1}{c\left(f_{0}\right)}+\alpha \tan \left(\frac{\pi y}{2}\right)\left[|f|^{y-1}-\left|f_{0}\right|^{y-1}\right]
$$

$\mathrm{f}$ and fo represents frequencies of analysis, $\mathrm{a}$ and $\mathrm{y}$ the two parameters modelizing the power law dependence of the attenuation. Analysis of the shear wave propagation allows deduction of $\mathrm{c}(\mathrm{fo}), \mathrm{a}$ and $\mathrm{y}$ enabling full rheological characterization of the tissue. In such cases, the estimation of group velocity values gives a representation of medium viscoelasticity at the central frequency of the wave spectral content.

- In a thin medium that has geometrical characteristics much smaller than the shear wavelength, such as arteries, guidance of the shear waves through medium leads to geometrical dispersion effects. Depending on the medium external environment and its geometrical characteristics, shear wave velocity propagation can be modeled and Young modulus can be deduced. The formula for a cylindrical artery can be written (Couade, 2010):

$$
v=\sqrt{K \frac{\omega h c}{\sqrt{3}}}
$$

Where $\mathrm{c}$ is the shear wave speed in an infinite medium, $\omega$ the frequency, $\mathrm{h}$ the height of the artery and $\mathrm{K}$ a correction factor. Young's modulus can then be deduced using the formula (6).

\subsection{A new imaging mode in ultrasound imaging \\ 3.2.1 Presentation}

Basic concepts introduced in the Supersonic Shear Imaging technique (Bercoff, 2004) have been used to create a real time imaging mode, called ShearWave Elastography (SWE), on a commercially available system. Two key aspects have made this innovation transfer possible:

- An new technology (multicore CPU, GPUs, as explained above) for the building of a ultrafast imaging system able to image transient shear waves. 
- A user workflow enabler: using this approach, the generation and imaging of the shear wave is performed with the same ultrasound probe as the one conventionally used for other imaging modes. No additional material is necessary to perform elasticity imaging. Acceptance of the mode in the clinical workflow is easier and the learning curve for the new mode is minimized.

The SWE mode is an additional real-time imaging mode that provides tissue elasticity estimation in kiloPascal (Fig. 12).

In its primary implementation, the mode estimates the shear wave group velocity locally and deduces the value in $\mathrm{kPa}$ assuming the medium is purely elastic (eq 6). This is done in real time on a 2D image plane as illustrated on figure below.

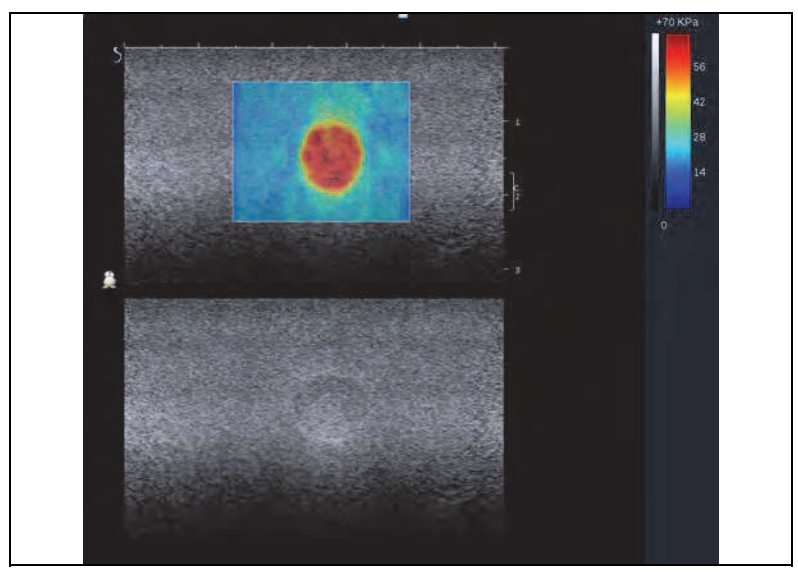

Fig. 12. ShearWave Elastography mode: Elasticity Information is displayed in real time in a box with color coded values.

Recently, the mode has been implemented on a specific probe providing volumetric imaging. In addition to axial view, transverse and coronal views of lesions elasticity distribution can be assessed as illustrated on figure below:

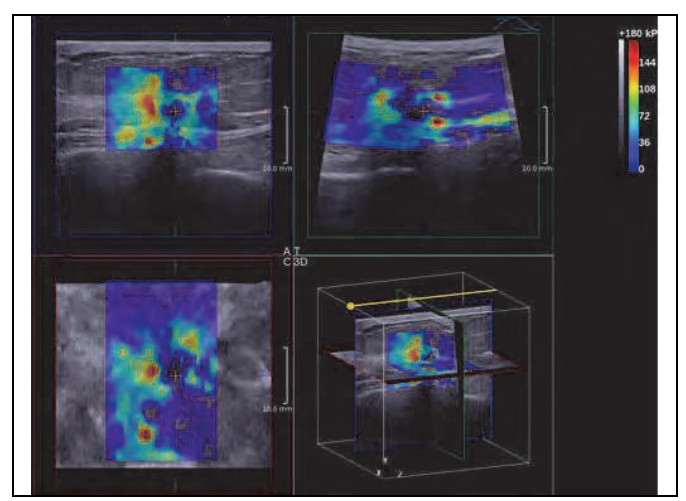

Fig. 13. 3D ShearWave Elastography. 3 planes can be viewed. By navigating within the volume the displayed imaging plane can be chosen. 
Volume assessment can be performed allowing more accurate visualization and quantification of elastic distribution. 3D SWE may be extremely useful in the framework of therapy monitoring.

Indeed complementary information of tissue stiffness changes can increase information on response to therapy compared to simple tumor volume size. Additional modules for specific applications requiring more complex rheological modelizations can be envisioned. In the example below, it is possible to measure and display the phase velocity as a function of the wave frequency in a region of interest of the liver. In addition to the group velocity, the slope of the phase velocity variation can be assessed. This full assessment of the liver viscoelasticity could be of great interest for diffuse liver disease diagnostics and staging.

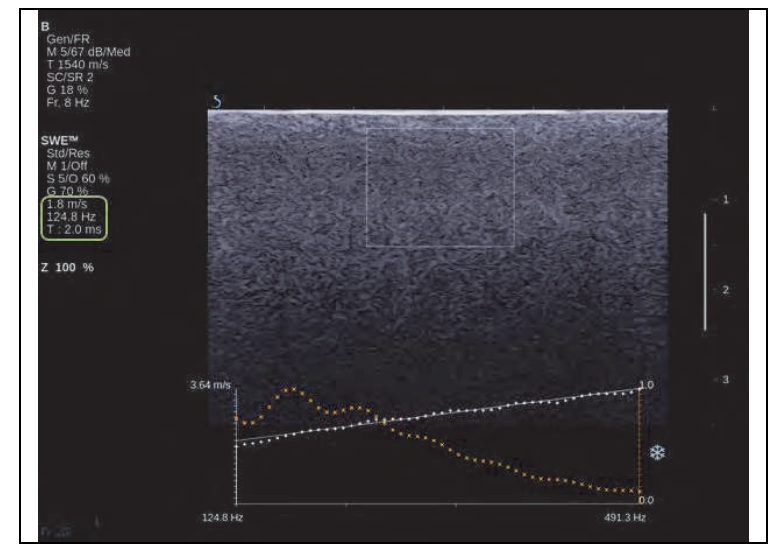

Fig. 14. Dispersion of shear waves in liver assessed by ShearWave Elastography. The white curve represents the shear wave phase velocity as a function of the frequency calculated in the square box displayed on the gray scale image. The yellow one is the spectrum amplitude of the transient wave. The group velocity, the central frequency of the shear wave and the relaxation time (in ms) corresponding to the slope of the curve are displayed to the left of the image.

The same principle can be applied to reconstruct the arterial Young's modulus by combining the group velocity measurement and the thickness of the artery as described in (8).

\subsubsection{Clinical value}

The ShearWave Elastography (SWE) mode has recently been implemented for different organs. Fig. 15 shows elasticity images in the breast, tendons, liver and prostate.

Multicentric studies to assess the clinical value of the mode are currently ongoing:

- Breast: SWE could potentially help improve the diagnosis of breast lesions by increasing the overall accuracy of the BI-RADS ${ }^{\circledR}$ (Mendelson, 2001) classification. Recent studies have shown promising results for breast cancer diagnostic (Evans, 2010). SWE could help correctly reclassify malignant lesions that would have been missed with ultrasound alone and declassify benign lesions that would have been biopsied. From a global perspective this could lead to a reduction of the number of unnecessary biopsies and an increase in positive biopsy rate reducing healthcare cost and patient's stressful experience.

A multicentric study on 1800 patients and 17 clinical sites is ongoing to confirm these preliminary results. 


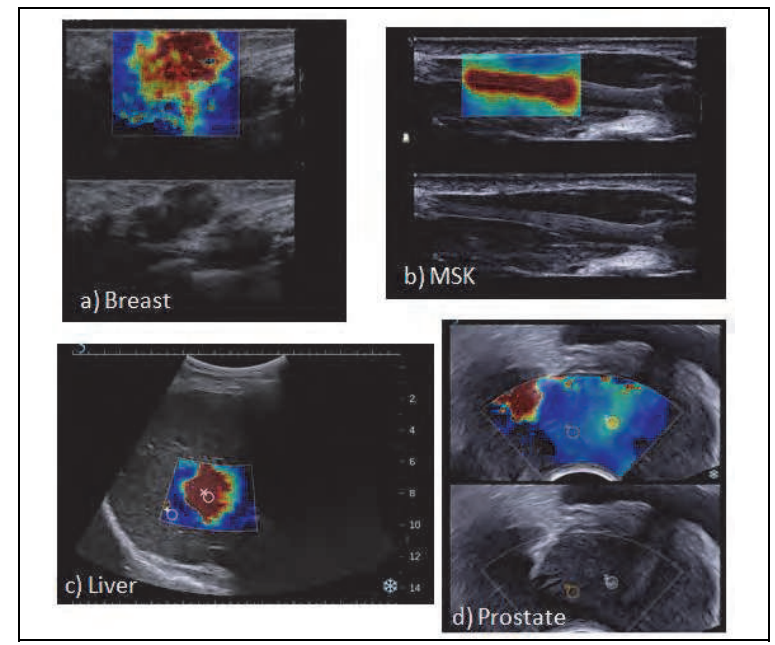

Fig. 15. SWE images in a) breast, b) MSK, c) liver, d) prostate

- $\quad$ Liver: SWE is currently being evaluated in the framework of diffuse liver disease staging and on its ability to improve the diagnosis of focal lesions. A preliminary study on 113 patients (Bavu, 2011) demonstrated high accuracy of fibrosis staging using SWE.

- Thyroid: As for breast, SWE could increase the diagnosis accuracy . The number of benign nodules that are undergoing Fine Needle Aspiration is currently extremely high. A preliminary study (Sebag, 2010) demonstrated very promising results on the pertinence of elasticity images for nodule characterization. More importantly, the specific case of follicular neoplasm raises an important issue as $85 \%$ of surgeries of this type of lesions are done for benign lesions.

- Prostate: SWE could be of great interest for improved detection rate of prostate cancer, through localization of prostate lesions, monitor localized treatments. Ongoing studies intend to demonstrate the value of SWE for increased positive biopsy rate in the prostate diagnostic workflow.

\subsubsection{Perspectives for SWE}

Today, SWE is mainly positioned in the disease diagnosis of specific static organs (cited above). However, given its specificities, other domains could benefit from the mode:

- Its resolution (up to $1 \mathrm{~mm}$ in superficial organs) and sensitivity (elastic contrasts of $20 \%$ can be detected) could improve screening for specific organs.

- Its quantitative aspect, implemented on a 3D imaging system, can target the emerging field of localized and minimally (or non) invasive therapy monitoring (RF, Cryoablation, HIFU).

- Its ability to acquire the elasticity information on a wide region of interest in a few tens of milliseconds allows elasticity imaging of moving organs and the analysis of elasticity variation in time. Cardiology is one of the areas where SWE could bring tremendous clinical value. Preliminary studies demonstrated that SWE could be a reliable and easy tool to assess heart myocardial stiffening (Couade, 2011). 


\section{Changing the paradigm in blood flow analysis using ultrafast imaging}

We demonstrated how ultrafast imaging could bring new information to the medical community by imaging fast transient phenomena such as shear waves. Ultrafast imaging can also be used to re-think conventional ultrasound modes. We will investigate in this section the potential of ultrafast imaging for flow analysis.

Doppler analysis is one of the most demanding features in ultrasound from a technical standpoint - the number and complexity of firings to acquire the information is huge - and from a performance standpoint - quantitative measurements are expected, raising the requirements of the mode in term of accuracy and reproducibility. Due to this complexity, Doppler tools suffer from technical limitations that impact the user in a significant way. It is shown here how ultrafast imaging can overcome those limitations and open new perspectives in Doppler analysis both in terms of performance and user workflow.

\subsection{Ultrafast sequence for Doppler imaging}

The same type of ultrafast sequences as the ones described above for B-mode (2.4) are used in Doppler imaging. Several tilted plane waves are sent into the medium and backscattered echoes are coherently summed to reconstruct ultrasound images. Then Doppler processing can be performed as on conventional images.

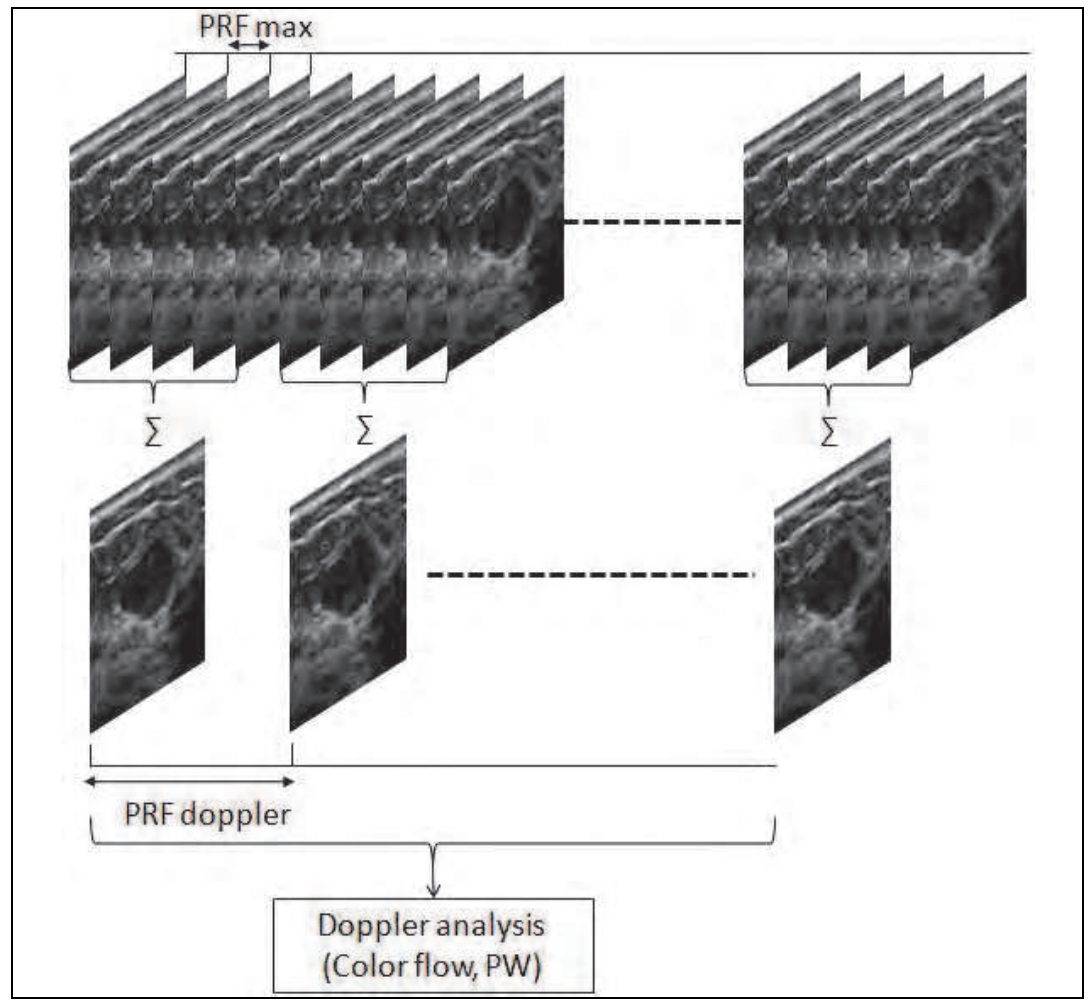

Fig. 16. Ultrafast compound imaging for Doppler analysis of flow. 
The maximum number of angles that can be used to compute an image is limited by the acquisition Pulse Repetition Frequency ( $\mathrm{PRF}_{\text {doppler }}$ ) needed to measure the desired Doppler velocity scale (usually this value is set by the user).

$$
\text { Nangles }=\frac{P R F_{\max }}{P R F_{\text {doppler }}}
$$

Where PRFmax is the maximal PRF reachable given the imaging depth considered.

Interestingly, it has been shown, in Color flow imaging, that resolution and sensitivity equivalent to classical schemes can be obtained using only 9 different angles (Bercoff, 2011). This indicates that Doppler images can be acquired 10 to 15 times faster than with conventional approaches (For a typical 20 to $30 \mathrm{~mm}$ color box, around 100 lines are acquired). Such a huge gain in acquisition time can be used in several ways:

- Increase Doppler imaging modes performance: we demonstrate below increases in temporal resolution and sensitivity (4.2).

- Improve user workflow: this gain in time can be used to perform other acquisition types such as PW, potentially increasing performances of highly demanding modes such as triple mode (Bmode, Color flow imaging and PW simultaneously).

- Change Doppler mode paradigm by merging Color flow imaging and PW Doppler modes in a single acquisition and with this, increase the accuracy of the examination and reduce its overall time (4.3).

The use of several tilted plane compounded waves as described above is essential for the performance of the mode in terms of sensitivity and accuracy (Udesen, 2008).

\subsection{Improving color flow imaging (CFI)}

Conventional schemes offer limited frame rates for color flow imaging (typically $20 \mathrm{~Hz}$ ) and suffer from severe trade-offs between image size and frame rate (that can go down to a few Hertz for boxes covering the whole image area). Ultrafast compound based Doppler imaging provides flow images with a temporal resolution never reached before on ultrasound systems whatever the box size. Complex and fast flows can be visualized in a much finer way potentially leading to a more reliable diagnosis of cardiovascular diseases such as stenoses.

The figure below shows images of a color flow clip acquired at $200 \mathrm{~Hz}$ (around 10 times faster than the conventional mode). A histogram of the velocities in one sample volume is provided as a function of time. It demonstrates the ability to quantify flow with very high temporal resolution.

With such high frame rates as demonstrated above $(100-200 \mathrm{~Hz})$, the visualization of the color clip can be done after acquisition through a slow motion movie.

For slower flow imaging, high frame rates are not necessary. Ultrafast sequences can therefore be tuned to increase spatial resolution and sensitivity. In a sensitive ultrafast acquisition, as the PRF required to measure slow flows is lower, the number of angles to compute a color image is increased to calculate the Doppler frequency shift. Sensitive color images acquired on the thyroid of a healthy volunteer is shown below and compared to classical color flow imaging. Deep small vessels are detected only on the ultrafast compounded image.

Increasing the ensemble length can even further optimize sensitivity. The number of samples used to calculate the flow per pixel can be increased up to a factor 15. Ultrasensitive images can be obtained like the ones reported by Institut Langevin on the rat brain (Macé, 2010) and may be of great interest in many applications: functional imaging of the brain, imaging of tumor vascularization, obstetrics.... 


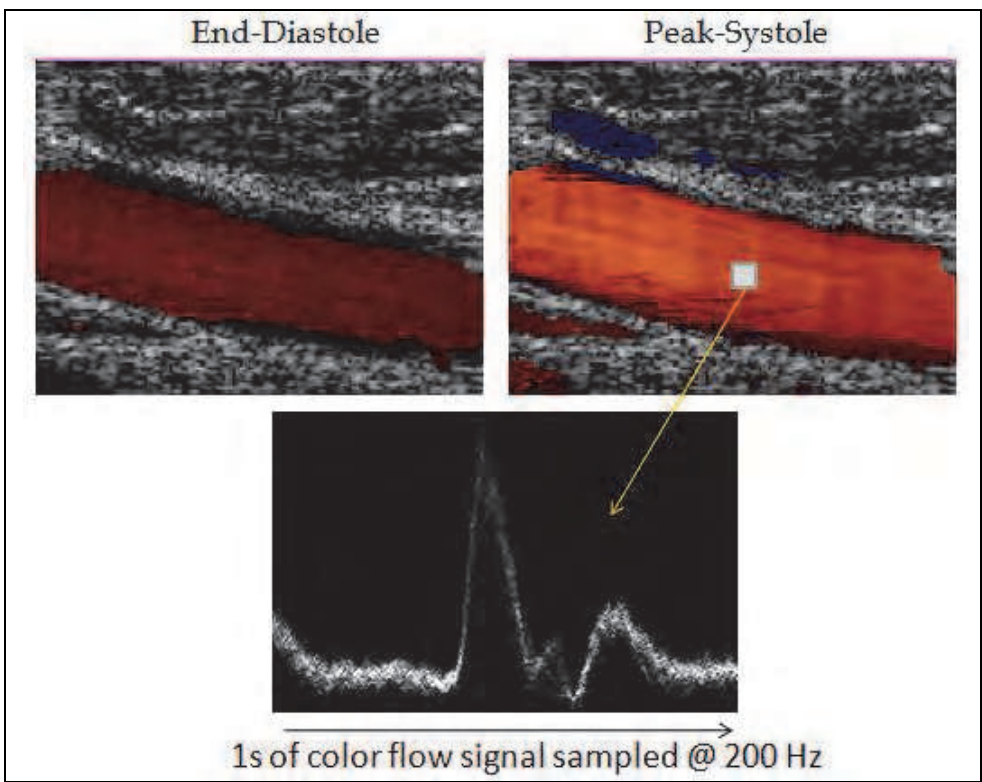

Fig. 17. Ultrafast color flow imaging provides very fine temporal resolution. The plot is computed by displaying a histogram of the velocity on the small ROI indicated as a function of time.

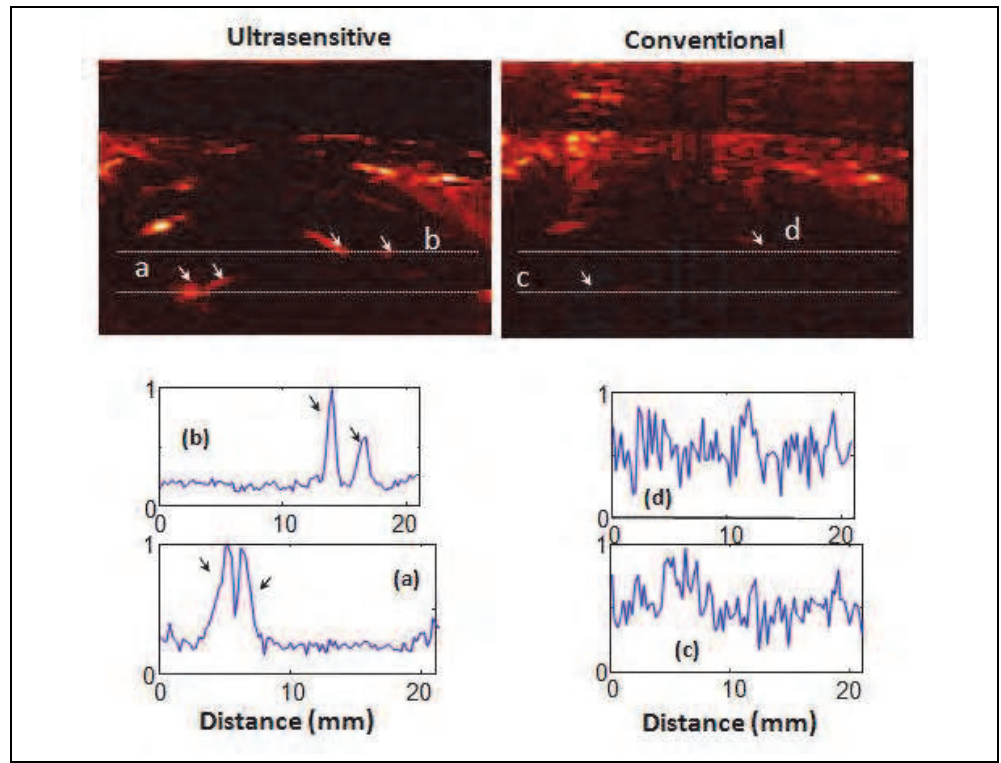

Fig. 18. Sensitive flow images based on ultrafast plane wave acquisition on the thyroid. Images have been acquired using 16 angles to compute ultrasound images and an ensemble length of 16 to deduce flow images. 
From a general perspective, ultrafast imaging breaks usual limitations and compromises of color flow imaging:

- Clips of color data can be generated with higher sensitivity and frame rate than on conventional systems.

- The increase quality is maintained whatever the box size. Usual schemes suffer from trade-offs between frame rate and color box size. Using plane waves, the whole area of interest can be filled with color Doppler information without any drop in frame rate.

The information is consistent and synchronous all over the imaged area. Doppler pixels have been assessed at the same time on the contrary of focused strategies where lines are sequentially acquired. In a classical approach, the Doppler signals on the sides of the box are therefore acquired with a time lag that can reach several hundreds of milliseconds.

\subsection{Quantitative ultrafast Doppler imaging}

Conventional Doppler analysis is usually performed using in two ultrasound modes:

- $\quad$ the color flow imaging mode to spatially locate a region of interest

- $\quad$ the Pulsed Wave mode to perform quantitative measurements at the region of interest depicted by color flow imaging. PW mode is a local assessment of quantitative information - information is assessed at one single location at a time.

In clinical exams, the user constantly goes back and forth between those two modes and successively analyzes with the PW the locations pointed out by the color flow imaging mode. Triple mode (simultaneous color and PW) has been introduced to improve the user workflow. Despite some compromises on the PW spectrum quality, it facilitates the acquisition of information in many cases.

Using an ultrafast architecture, Doppler can be envisioned in a completely different manner: quantitative information is acquired at the same time in all pixels of the color box breaking the incompatibility between imaging and quantitative measurements. In a typical implementation, a one shot acquisition can be launched from the conventional color flow imaging mode. A full clip of Doppler data is acquired (typically 2 to $6 \mathrm{~s}$ ) and the system is frozen. The user can then review the color flow imaging clip, locate the frame of interest that better depicts flow properties and perform PW measurements at several locations, allowing for the first time a comparison of spectra from different regions of interest from the same cardiac cycle.

The quantitative ultrafast Doppler acquisition workflow is illustrated on the figure 19.

Using the retrospective review of ultrafast data, many automatic tools can be added to help physician diagnosis:

- Comparison of flow data from several locations (as described above)

- Automatic localization of peak velocities within the image for accurate flow quantification

- Calculation and display of the mean and peak velocities all over the image

- Automatic calculation and compensation of the Doppler angle.

Ultrafast imaging opens perspectives to Doppler imaging by enhancing its performances, allowing visualization of very fast flow characteristics, perform accurate quantification and comparison of flow velocities through the whole image area and provide new types of 
visualization and automation tools. It will probably allow a significant reduction of the vascular exam duration as all data necessary for the diagnosis of a given area is acquired in a few seconds.. In the future, ultrafast Doppler could be used to derive new information such as the shear stress on the arterial wall, perform accurate vortex analysis and quantification

\section{Conclusion}

ShearWave Elastography and Ultrafast Doppler are two important innovations that are made possible thanks to ultrafast imaging. They are the first demonstrations of many other benefits that an ultrafast architecture can bring to the clinical world. Cardiovascular is one of the fields that could tremendously benefit from this technology. Ultrafast imaging can change the way moving organs are imaged. Recent works demonstrated, for example, the ability to locally measure Pulse Wave Velocity in the artery in less than one second (Couade 2010). Other publications reported dynamic analysis of heart mechanics using SWE for higher performance and more accurate detection of cardiovascular diseases. Elasticity of myocardium has been assessed locally as a function of time through a whole heart cycle (Couade 2011). Ultrafast imaging can also provide analysis of other transient phenomena that have not been extensively explored such as the dissolution of ultrasound contrast agents (Couture 2009) or the monitoring of brain activity (Macé 2010) through ultrasensitive flow images - positioning for the first time ultrasound in the field of functional brain imaging.

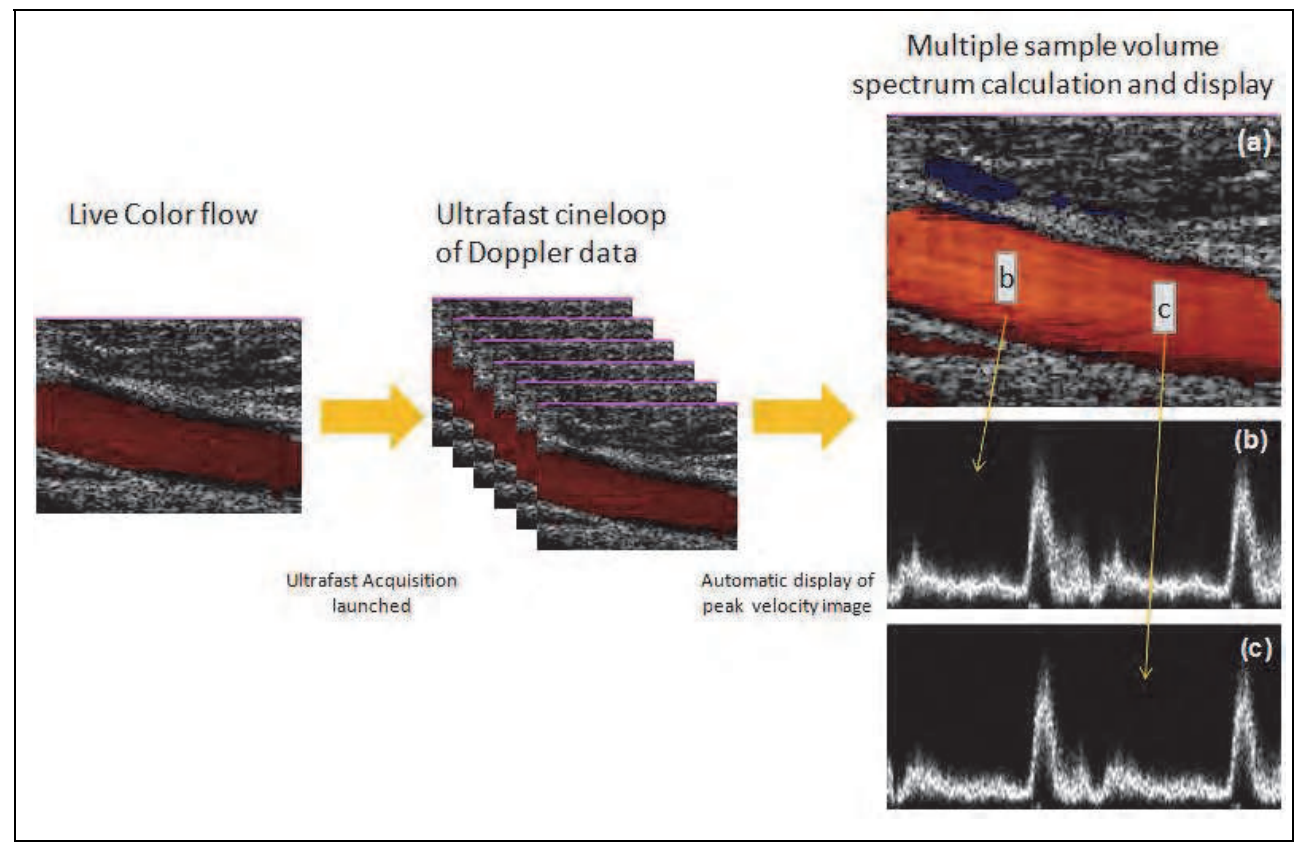

Fig. 19. Doppler analysis workflow.

There is no doubt that such ultrafast imaging based academic works will create new standards in ultrasound imaging in the next coming years - in 2D, as well as, on a longer perspective, in $4 \mathrm{D}$. 


\section{Acknowledgment}

I would like to thank Jessica Bercoff, Claude Cohen-Bacrie, Aline Criton, Michèle Debain and Jacques Souquet for their support, feedback and enthusiasm on this chapter as well as all scientific contributors of the reported work (Matt Bruce, Mathieu Couade, Nicolas Felix, Mathias Fink, Christophe Fraschini, Jean Luc Gennisson, Fabien Mezière, Emilie Macé, Gabriel Montaldo, Thanasis Loupas, Mathieu Pernot, David Savéry, Mickael Tanter ....)

\section{References}

Bavu, E.; Gennisson,J.L.; Couade, M.; Bercoff, J.; Mallet, V.; Fink, M.; Nalpasc, B.; Tanter, M.; Pol, S. (2011). Non-invasive in-vivo Liver Fibrosis evaluation using Supersonic Shear Imaging: a clinical Study on 113 Hepatitis C Virus patients. accepted for publication in Ultrasound in Medicine and Biology.

Bercoff, J.; Tanter, M.; Fink, M. (April 2004). Supersonic shear imaging: A new technique for soft tissues elasticity mapping, IEEE Transactions on Ultrasonics, Ferroelectrics and Frequency Control, vol. 51, no. 4, pp. 396-409.

Bercoff, J.; Montaldo, G.; Loupas, T.; Savery, D.; Mézière, F.; Fink, M.; Tanter, M. (January 2011). Ultrafast compound Doppler imaging: providing full blood flow characterization, IEEE Transactions on Ultrasonics, Ferroelectrics and Frequency Control, 58(1), pp. 134-47.

Catheline, S.; Wu, F.; Fink M. (May 1999). A solution to diffraction biases in sonoelasticity: the acoustic impulse technique. J Acoust Soc Am, 105(5), pp. 2941-50.

Christensen, C. M. ( 2003). The Innovator's Dilemma, HarperBusiness Essentials, 2003

Couade, M.; Pernot, M.; Prada, C.; Messas, E.; Emmerich, J.; Bruneval, P.; Criton, A.; Fink, M.; Tanter, M. (October 2010). Quantitative assessment of arterial wall biomechanical properties using shear wave imaging, Ultrasound Med Biol., 36(10), pp. 1662-76.

Couade, M.; Pernot, M.; Messas, E.; Bel, A.; Ba, M.; Hagege, A.; Fink, M.; Tanter, M. (February 2011). In vivo quantitative mapping of myocardial stiffening and transmural anisotropy during the cardiac cycle. IEEE Trans Med Imaging. 2011 Feb, 30(2), pp. 295305.

Couture, O.; Bannouf, S.; Montaldo, G.; Aubry, J.F.; Fink, M.; Tanter, M. (November 2009). Ultrafast imaging of ultrasound contrast agents. Ultrasound Med Biol, 35(11), pp. 1908-16.

Delannoy, B.; Torgue, R.; Bruneel, C.; Bridou, E. (1979). Ultrafast electronic image reconstruction device, Echocardiology, Vol. 1, C. T. Lancee, Ed., ch. 3, pp 447450.

Evans, A.; Whelehan, P.; Thomson, K.; McLean, D.; Brauer, K.; Purdie, C.; Jordan, L.; Baker, L.; Thompson, A. (2010). Quantitative shear wave ultrasound elastography: initial experience in solid breast masses. Breast Cancer Res. 12(6).

Jensen, J.A.; Holm, O.; Jerisen, L.J.; Bendsen, H.; Nikolov, S.I.; Tomov, B.G.; Munk, P.; Hansen, M.; Salomonsen, K.; Hansen, J.; Gormsen, K.; Pedersen, H.M.; Gammelmark, K.L. (May 2005). Ultrasound research scanner for real-time synthetic 
aperture data acquisition, IEEE Transactions on Ultrasonics, Ferroelectrics and Frequency Control, Volume 52, Issue 5, pp. 881 - 891

Lu, J-Y. (January 1998). Experimental study of high frame rate imaging with limited diffraction beams, IEEE Transactions on Ultrasonics, Ferroelectrics and Frequency Control, Volume 45, Issue 1, pp. $84-97$

Macé, E.; Montaldo, G.; Bercoff, J.; Cohen, I.; Fink, M.; Tanter, M. (2010). Ultrafast Doppler Imaging and its application to high sensitivity brain angiography. IEEE UFFC conference.

Mendelson, E.B.; Berg, W.A.; Merritt, C.R. (July 2001). Toward a standardized breast ultrasound lexicon, BI-RADS: ultrasound. Semin Roentgenol. 36(3), pp. 217-25.

Montaldo, G.; Tanter, M.; Bercoff, J.; Benech, N.; Fink, M. (March 2009). Coherent plane-wave compounding for very high frame rate ultrasonography and transient elastography, IEEE Transactions on Ultrasonics, Ferroelectrics and Frequency Control, 56(3), pp. 489-506.

Orescanin, M.; Qayyum, M.A.; Toohey, K.S.; Insana, M.F. (October 2011) Dispersion and shear modulus measurements of porcine liver. Ultrasonic Imaging, 32(4), pp. 255-66.

Rosenzweig, S.; Palmeri, M.; Nightingale, K. (February 2011). GPU-Based Real-Time Small Displacement Estimation With Ultrasound, IEEE Transactions on Ultrasonics, Ferroelectrics and Frequency Control, 58(2), pp. 399-405.

Royer, D.; Dieulesaint, E. (2000). Elastic Waves in Solids: Free and guided propagation, Springer.

Sandrin, L.; Catheline, S.; Tanter, M.; Vinçonneau, C.; Fink, M. (2000), 2D transient elastography, Acoust. Imaging, vol 25, pp. 485-492

Sarvazyan, A.P.; Rudenko, O.V.; Swanson, S.D.; Fowlkes, J.B.; Emelianov, S.Y. (November 1998). Shear wave elasticity imaging: a new ultrasonic technology of medical diagnostics. Ultrasound Med Biol. 24(9), pp. 1419-35.

Schiwietz, T.; Chang, T.; Speier, P.; Westermann, R. (2006). Mr image reconstruction using the GPU, Proc. SPIE, vol. 6142, no. 1.

Sebag, F.; Vaillant-Lombard, J.; Berbis, J.; Griset, V.; Henry, J.F.; Petit, P.; Oliver, C. (December 2010). Shear wave elastography: a new ultrasound imaging mode for the differential diagnosis of benign and malignant thyroid nodules. J Clin Endocrinol Metab. 95(12), pp. 5281-8.

Shattuck, D. P.; Weinshenker, M. D.; Smith, S. W.; Von Ramm, O. T. (April 1984). Explososcan: A parallel processing technique for high speed ultrasound imaging with linear phased arrays, The Journal of the Acoustical Society of America, Volume 75, Issue 4, pp.12731282

Szabo, T. L. (2004). Diagnostic ultrasound imaging: inside out, Elsevier Academic Press. Von Ramm, O.T.; Smith, S.W.; Pavy, H.G., Jr. (March 1991), High-speed ultrasound volumetric imaging system. II. Parallel processing and image display Ultrasonics, Ferroelectrics and Frequency Control, IEEE Transactions on Volume 38, Issue 2, pp.109 115

Szabo, T.L. (1994). Time domain wave equations for lossy media obeying a frequency power law, J. Acoust. Soc. Am. Volume 96, Issue 1, pp. 491-500.Xu, F.; Mueller, K. (2007). Realtime $3 d$ computed tomographic reconstruction using commodity graphics hardware, Phys. Med. Biol., vol. 52, no. 12, pp. 3405-3419. 
Udesen, J.; Gran, F.; Hansen, K. L.; Jensen, J. A.; Thomsen, C.; Nielsen, M. B. (August 2008). High Frame-Rate Blood Vector Velocity Imaging Using Plane Waves: Simulations and Preliminary Experiments, IEEE Transactions on Ultrasonics, Ferroelectrics and Frequency Control, vol. 55, no. 8, pp. 1729-1743

$\mathrm{Xu}, \mathrm{F}$; Mueller, K. (2007). Real-time $3 d$ computed tomographic reconstruction using commodity graphics hardware Phys. Med. Biol., vol. 52, no. 12, pp. 3405-3419 


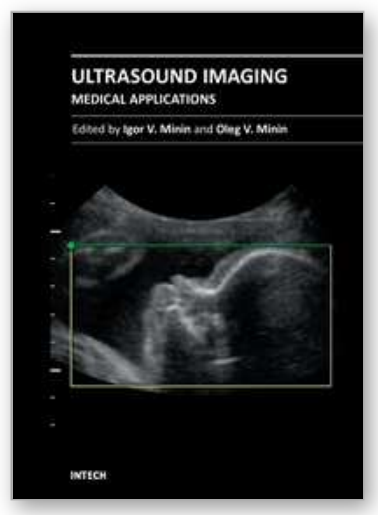

\author{
Ultrasound Imaging - Medical Applications \\ Edited by Prof. Oleg Minin
}

ISBN 978-953-307-279-1

Hard cover, 330 pages

Publisher InTech

Published online 23, August, 2011

Published in print edition August, 2011

This book provides an overview of ultrafast ultrasound imaging, 3D high-quality ultrasonic imaging, correction of phase aberrations in medical ultrasound images, etc. Several interesting medical and clinical applications areas are also discussed in the book, like the use of three dimensional ultrasound imaging in evaluation of Ashermanâ $€^{\mathrm{TM} S}$ syndrome, the role of 3D ultrasound in assessment of endometrial receptivity and follicular vascularity to predict the quality oocyte, ultrasound imaging in vascular diseases and the fetal palate, clinical application of ultrasound molecular imaging, Doppler abdominal ultrasound in small animals and so on.

\title{
How to reference
}

In order to correctly reference this scholarly work, feel free to copy and paste the following:

Jeremy Bercoff (2011). Ultrafast Ultrasound Imaging, Ultrasound Imaging - Medical Applications, Prof. Oleg Minin (Ed.), ISBN: 978-953-307-279-1, InTech, Available from: http://www.intechopen.com/books/ultrasoundimaging-medical-applications/ultrafast-ultrasound-imaging

\section{INTECH}

open science | open minds

\section{InTech Europe}

University Campus STeP Ri

Slavka Krautzeka 83/A

51000 Rijeka, Croatia

Phone: +385 (51) 770447

Fax: +385 (51) 686166

www.intechopen.com

\section{InTech China}

Unit 405, Office Block, Hotel Equatorial Shanghai

No.65, Yan An Road (West), Shanghai, 200040, China

中国上海市延安西路65号上海国际贵都大饭店办公楼 405 单元

Phone: +86-21-62489820

Fax: $+86-21-62489821$ 
(C) 2011 The Author(s). Licensee IntechOpen. This chapter is distributed under the terms of the Creative Commons Attribution-NonCommercialShareAlike-3.0 License, which permits use, distribution and reproduction for non-commercial purposes, provided the original is properly cited and derivative works building on this content are distributed under the same license. 